\title{
Lessons Learnt during the COVID-19 Pandemic: For Patients with End-Stage Renal Disease, We Should Prioritize Home-Based Treatment and Telemedicine
}

\author{
Giuliano Brunori $^{\mathrm{a}}$ Gianpaolo Reboldi $^{\mathrm{b}}$ Filippo Aucella ${ }^{\mathrm{c}}$ \\ aDivision of Nephrology and Dialysis, Santa Chiara Hospital, Trento, Italy; ${ }^{\text {bDepartment }}$ of Medicine, \\ University of Perugia, Perugia, Italy; 'Nephrology and Dialysis Unit, Fondazione "Casa Sollievo Della Sofferenza," \\ IRCCS, San Giovanni Rotondo, Italy
}

\section{Keywords}

Dialysis · Home treatment · Telemedicine · Coronavirus disease 2019

\begin{abstract}
Backgrounds: The recent coronavirus disease 2019 (COVID-19) pandemic has placed worldwide health systems and hospitals under pressure, and so are the renal care models. This may be a unique opportunity to promote and expand alternative models of health-care delivery in patients undergoing renal replacement therapies. Summary: Despite the high risk of acquiring communicable diseases when undergoing in-centre treatments, only a small proportion of patients are currently being treated with home therapies. Recent data provided by the Italian Society of Nephrology (SIN), the REIN French Registry and the Wuhan Hemodialysis Quality Control Center clearly show that patients receiving hospital-based treatment have a 3- to 4-fold greater risk of infection, and a subsequent fatality proportion between 21 and $34 \%$. On the other hand, home-based therapy can be managed remotely, there is little or no need for transport to and from the hospital, and it is less expensive. Besides, the digital revolution in health care with the development of vir-
\end{abstract}

karger@karger.com www.karger.com/kbr

Karger $\stackrel{\text { ' }}{5}$

BOPEN ACCESS
(C) 2021 The Author(s)

Published by S. Karger AG, Basel

This article is licensed under the Creative Commons AttributionNonCommercial-NoDerivatives 4.0 International License (CC BYNC-ND) (http://www.karger.com/Services/OpenAccessLicense). Usage and distribution for commercial purposes as well as any distribution of modified material requires written permission. tual care systems can make home dialysis with telehealth a cost-effective solution for both patients and health-care providers. Such a transition would require specific training for physicians and health-care professionals and a functional reorganization of dialysis centres to improve the skills and expertise in caring for patients at home. Conclusion: The need for more widespread home treatment is the main lesson learnt by nephrologists by the COVID-19 pandemic.

(C) 2021 The Author(s)

Published by S. Karger AG, Basel

\section{Introduction}

Over the past decades, viral outbreaks and their complex interactions with humans and animals have resulted in cross-species transmission, posing a great threat to human health and safety [1]. In recent years, there has been an upsurge in newly identified coronaviruses, such as the severe acute respiratory syndrome coronavirus (SARS$\mathrm{CoV}$ ) and the Middle East respiratory syndrome coronavirus. Last winter, a novel coronavirus named SARSCoV-2, initially identified in China, spread worldwide and in February 2020, the World Health Organization (WHO) named the disease "coronavirus disease 2019" 
(COVID-19). Shortly afterwards, the WHO declared COVID-19 a pandemic [2].

Many countries introduced lockdown restrictions along with the mandatory use of personal protective equipment, social distancing, and quarantine measures to minimize person-to-person contagion. Such restrictive measures determined substantial changes in many areas of society, while health systems and hospitals were overwhelmed by the spread of the infection and its deadly consequences. Thus, the COVID-19 pandemic represents a unique opportunity to promote and expand alternative models of health-care delivery.

Home care programs and telemedicine can ensure the same high efficacy standards and outcomes as that typically provided by health-care facilities and hospitals. Renal patients, especially those on dialysis, are often elderly and have comorbid conditions (e.g., diabetes, hypertension, chronic obstructive pulmonary disease, heart disease), placing them at very high risk for COVID-19 [3].

In many countries, dialysis is predominantly delivered as in-centre haemodialysis (ICHD), while a minority of patients undergo dialysis at home, either as home haemodialysis (HD) or peritoneal dialysis (PD) [4]. Such choice is somewhat puzzling since several reports have shown comparable, if not better, outcomes in home-treated patients compared to those receiving ICHD $[5,6]$. It is well known that patients receiving ICHD are at high risk of acquiring communicable diseases, such as viral hepatitis (HBV or HCV), that still remains an issue, along with bacterial colonization of water and catheters, a major reason of concern in dialysis units $[7,8]$.

\section{Lessons Learnt during the COVID-19 Pandemic}

On any given weekday, patients with ESRD undergo ICHD, a life sustaining and unavoidable treatment, in crowded facilities. Transport to the dialysis centre exposes patients to drivers and fellow patients, sometimes for hours, depending on the distance to be covered, thereby increasing the risk of cross-infection and contagion. Patients then remain in HD rooms in close contact with other fellow patients, nurses, technicians, and nephrologists for as long as $4-5 \mathrm{~h}$. Thus, dialysis units may become a high-risk environment where airborne viral pathogens such as SARS-CoV-2, transmitted through droplets and contact, can find a favourable milieu to spread the infection. A number of specialized health-care workers must, therefore, operate in such a hazardous environment. Consequently, because of illness or quarantine, the actual workforce capacity can drop below critical thresholds for safe and effective HD delivery. In the era of the COVID-19 pandemic, maintaining the necessary workforce can be challenging. Nephrology nurses, for instance, are essential for dialysis treatment and, in case of illness or quarantine, substitutes may not be readily available. During the pandemic, several centres had to reduce dialysis frequency from 3 to 2 times per week, and duration from 4 to $3 \mathrm{~h}$ due to the scarcity of dialysis nurses.

What should we do to prevent COVID-19 or other future pandemics that could affect patients, physicians, and health-care professionals? Broader adoption of home treatments is perhaps an available and effective answer [9-11].

With a reduced schedule of facility access and a higher likelihood of adherence to general prevention measures, such as personal protective equipment, social distancing, and lockdown restrictions, patients on homebased therapy can reduce their risk of infection. This emerges from the findings of a cross-sectional survey carried out by the Italian Society of Nephrology (SIN). The e-survey questionnaire was sent by e-mail on April 9, 2020, and had to be returned by April 23, 2020; it allowed us to evaluate the impact of SARS-CoV-2 infection in patients on renal replacement treatment (RRT) during the exponential phase of the pandemic [12]. All 454 nephrology centres in Italy received the questionnaire, and by the deadline date, 365 centres had responded ( $80.4 \%$ response rate). The surveyed RRT population included 30,821 HD, 4,139 PD, and 25,481 kidney transplant (Tx) patients. There were 1,368 SARS-CoV-2-positive patients (1,093 in HD, 57 in PD, and 218 in Tx), with an overall $2.26 \%$ positivity rate among the 60,441 patients on RRT who were surveyed $(30,821$ in $\mathrm{HD}, 4,139$ in PD, and 25,481 in Tx). The proportion of positive patients was significantly higher in $\mathrm{HD}$ than that in $\mathrm{PD}$ or $\mathrm{Tx}$ (3.55\% in $\mathrm{HD}, 1.38 \%$ in $\mathrm{PD}$, and $0.86 \%$ in $\mathrm{Tx} ; p<0.001$ ). The fatality proportions in infected patients were $34 \%$ in HD (369/1,093), $46 \%$ in PD (26/57), and $25 \%$ in $\mathrm{Tx}$ (54/218). The absolute number of deaths was indeed higher in HD, but the infection fatality proportion was broadly comparable across the 3 groups, thereby suggesting that even at low infection rates, as observed in PD and in $\mathrm{Tx}$, fatality is frequent in SARS-CoV-2 positive RRT patients as a whole. It does not matter whether patients are treated in hospital-based dialysis facilities or at home; if they test positive, the fatality proportion is similar. These data emphasize the importance of containing infection spread even when the absolute number of positive cases is low as in PD or in Tx patients.
Brunori/Reboldi/Aucella 
A retrospective analysis of the online registration system of the Wuhan Hemodialysis Quality Control Center found that out of 7,154 registered patients, 154 had laboratory-confirmed COVID-19 between January 1 and March 10, 2020 [13]. The registration system updated the information of confirmed and suspected COVID-19 patients from 65 medical institutions. After excluding 23 patients for whom verbal consent was not available, 131 lab-confirmed cases were included in the final analysis. By the study cut-off date, 47 patients were alive and had been discharged, 43 were alive but still hospitalized, and 41 patients had died. The fatality proportion in this group was $31 \%$, which is fairly consistent with the figures reported by the Italian survey.

Recently, the French REIN Registry published national data on the incidence of COVID-19 in dialysis patients, the course of the illness, and the risk factors associated with mortality [14]. The French national cohort consists of 48,669 dialysis patients treated in 1,245 dialysis units in metropolitan France and overseas territories. A total of 1,621 infected patients were reported in the REIN registry from March 16 to May 4, 2020. Among them, 344 died due to a SARS-CoV-2-related cause after a median time of 6 days, an infection fatality proportion of $21 \%$. The probability of being affected was higher for males, diabetic patients, and those requiring assistance for transfer or being treated in a self-care unit. Univariate analysis showed that older age, being a former smoker, having a chronic respiratory disease, cardiovascular comorbidities, and frailty (hypoalbuminaemia or inability to walk) were associated with a higher risk of death in SARS-CoV2-infected dialysis patients. Dialysis in self-care units or out-centres, or being a current smoker was associated with a lower risk of death. As a matter of fact, most of these clinical characteristics and treatment modalities were associated with older age. Interestingly, dialysis at home was also associated with a lower probability of being infected. As per the SIN survey [12], SARS-CoV-2 positivity showed substantial heterogeneity ranging from $<1$ to $10 \%$ among regions.

Taken together, these findings show that patients on hospital-based treatment have a 3- to 4-fold greater risk of infection and a subsequent fatality proportion between 21 and 34\%. Hence, given the lower risk of SARS-CoV-2 infection with home-based treatments compared with ICHD, having larger proportions of patients in home programs, or more transplants, will limit the infection spread among vulnerable patients and likely reduce the disease and health-care burden of COVID-19. Home treatments (dialysis or transplant) usually require 1 visit

COVID-19, Home-Based Treatment and Telemedicine per month, and most visits can be done via telemedicine, rather than in-person visits 3 times a week as is the case with HD patients.

The recent epidemic highlighted some of the limits of hospital-based treatment. Dialysis centres and patients were hit dramatically, and although specific protocols and recommendations were soon developed and adopted, the absence of effective and evidence-based therapeutic agents left nephrologists substantially unarmed. Nowadays, emerging clinical evidence supports the use of antivirals, dexamethasone, and immune-based therapy for hospitalized patients with COVID-19 who require supplemental oxygen, but there are no drugs or other therapeutics presently approved to prevent COVID-19. In the absence of specific agents, the efficacy of adjunctive therapies is under investigation [15]. Several studies support the use of anticoagulants, and current guidelines include thrombosis prophylaxis with low-molecular-weight heparin (LMWH) for COVID-19 patients. Beyond such indication, recent observations suggest that LMWH, a commonly used anticoagulant during HD sessions, might have protective effects on virus entry into the host cells, thereby reducing the infection burden and its consequences [16]. Regrettably, at the time of this writing, sound clinical evidence supporting such intriguing preventive role of LMWH in HD patients is still lacking.

Even though large units can ensure economies of scale and stringent standardized policies [17], they carry an inherent risk of contagion and infection spread. On the contrary, home-based treatment may have distinct advantages: (a) patients can be managed remotely; (b) less demand for transport to and from the hospital (1 every 4-5 weeks for PD vs. 13 per month for HD) and consequently a positive impact on the environment (by reducing pollution from fossil fuels); (c) lower costs in many countries [4]. Obviously, we have to bear in mind that all that glitters is not gold. The advantages of home dialysis can be nullified by difficulties in dialysis supplies delivery due to strained supply chains. In such instances, for stable patients, the volume exchange can judiciously be reduced, thereby allowing supplies to last longer. Moreover, by using remote supervision and monitoring, the team can maintain close contact and guide patients and caregivers until a proper solution is found. Incidentally, remote patient monitoring systems are already available for automated PD cyclers, and the COVID-19 pandemic has accelerated the transition to virtual care [18-20].

The COVID-19 pandemic boosted the digital revolution in health care [21] and moved the traditional hospital-based visits into a virtual care environment. Telemed- 
icine is an intervention that has been implemented occasionally in dialysis care in the past event though it offers additional risk reduction and other benefits for patients, staff, and providers. Using telehealth, we can limit physical congregation in facilities and maintain appropriate "social distancing." Nephrologists and staff can ensure safe and effective ICHD, while patients at home can "dial in" to telehealth visits and maintain continuity of care. All health-care workers, including dialysis nurses, dieticians, and social workers, can participate in virtual care programmes and sessions, thereby limiting their own risk of exposure. Early in the course of the pandemic, public and private stakeholders began planning and implementing a variety of telehealth interventions designed to reduce SARS-CoV-2 exposure and spread. Commercially available systems can be adapted to dialysis requirements although integrating telehealth into an existing practice requires careful planning with staff, regulatory and legal officers, and health authorities. Telemedicine must include secure communications, realtime messaging, telephony or video conferencing, and patient and device monitoring. Virtual care systems employing integrated electronic medical records with realtime data collection, and decision support tools, can improve active surveillance and timely case finding identification. Remote monitoring and assessment will also allow medication review, education, and support for patients and caregivers and coordination between healthcare team members [20]. Besides, patients on home dialysis may benefit from daily vital signs and physiologic parameters monitoring to guide on-going adjustments in treatment plans, and information technology can indeed make it easier for both patients and physicians. Prospectively, integrated virtual health systems can increase patient empowerment and independence in home treatment while still maintaining safety standards, facilitate contact with residents in remote areas, and make home dialysis with telehealth a cost-effective solution for both patients and health-care providers by eliminating transportation-related costs.

Although the vast majority of home patients can effectively be managed remotely, there are patients who require in-person visits to the PD units. These include patients in training, patients on urgent start PD, and patients with acute conditions such as peritonitis. For these patients, a "call-first" policy can be implemented to limit time spent in waiting rooms. Telehealth is an important tool in the evaluation of these patients before they actually enter the nephrology unit, especially if patients have symptoms or a positive history for SARS-Cov-2 exposure.
Unfortunately, in the midst of a crisis, a rapid shift from hospital-based treatment to home-based therapies cannot be carried out easily. Such a transition would require specific training for physicians and health-care professionals and a functional re-organization of dialysis centres to improve the skills and expertise in caring for patients at home and not in hospital. Straightforward technology and simplified bureaucracy are needed to smooth the learning curve and facilitate the implementation of assistance networks (i.e., assisted dialysis for patients without caregivers or for nursing home patients). New patients at home should be preferentially placed on continuous ambulatory peritoneal dialysis (CAPD) because the training for APD is more timeconsuming and, especially for elderly patients, more complex. APD is also more frequently associated with drain alarms and lost dwells, in particular, during the first weeks of treatment when the ultrafiltration capacity of the peritoneum needs to be tested. This often requires medical interventions and/or machine replacement, thereby involving patients, staff, and supply chains.

Dialysis prescription changes for PD patients or home HD patients should be made as needed based on the patient's clinical status and laboratory exams. Non-essential tests, such as the peritoneal equilibration test, can be safely postponed in the short term if the patient remains clinically stable. Deferral of non-essential testing is recommended by the International Society for Peritoneal Dialysis and the UK Renal Association [22]. All routine oral medications should be prescribed to ensure coverage for a 90-day period. In order to avoid any problems with medical supply chains that could affect delivery of supplies to the home dialysis patient, we recommend that our patients should keep an 8-week stock of fluids and medications on hand. Prior to the COVID-19 period, patients were allowed to have a 5-week stock of supplies at home (the interval period between 1 medical visit and the next). In addition, we advise our patients to use either homemade cloth masks or surgical masks during connection and disconnection (both in PD and in HD).

The delayed response to COVID-19 in many countries, in part due to the initially limited knowledge and underestimation of the potential disease burden, highlights the lack of planning and preparedness. The late response is indeed an issue because many experts and scientists issued warnings in order to anticipate a viral pandemic. Patients on dialysis are vulnerable and at high risk of infection in hospital-based treatments. In particular, ICHD is a treatment relying on old schemes and thoughts, which originated in the late 1970s, but that is still the most widely method by tens of thousands of patients around the world. 
Table 1. Comparison of home dialysis and in-centre dialysis programmes

\begin{tabular}{|c|c|c|}
\hline & Home dialysis & In-centre dialysis \\
\hline Contact & $\begin{array}{l}\text { As low as } 1 \text { per month in-person visit and contact with HCWs. Patients } \\
\text { can be monitored and managed using telemedicine }\end{array}$ & $\begin{array}{l}\text { Al least } 13 \text { per month in-person visits and close contacts } \\
\text { with HCWs and other patients }\end{array}$ \\
\hline Workforce & No need of in-person HCWs' support & $\begin{array}{l}\text { HCWs are required } \\
\text { Risk of shortage of qualified personnel due illness or } \\
\text { quarantine }\end{array}$ \\
\hline Resources & $\begin{array}{l}\text { Supply chain might have problems due to lockdown restriction, but } \\
\text { with a low number of patients in home dialysis programmes the actual } \\
\text { shortage risk is low }\end{array}$ & $\begin{array}{l}\text { A large quantity of protective personal equipment is } \\
\text { required. Supply chain could face problems due to the } \\
\text { large number of centres and patients to be supplied }\end{array}$ \\
\hline Transport & $\begin{array}{l}\text { Flexible schedule with low number of trips per year. Positive impact on } \\
\text { the environment due to reduced fossil fuel consumption }\end{array}$ & $\begin{array}{l}\text { Pre-programmed rigid schedule and logistics } \\
\text { Negative impact on the environment from fossil fuels } \\
\text { consumption. Need for parking space close to the dialysis } \\
\text { unit }\end{array}$ \\
\hline Family & $\begin{array}{l}\text { Very often the family members are involved in treatment management } \\
\text { and patient care. In some instances, the caregiver burden may be } \\
\text { overwhelming for family members }\end{array}$ & $\begin{array}{l}\text { HCWs carry out in-centre dialysis with no involvement of } \\
\text { the family }\end{array}$ \\
\hline Infection spread & The risk of infection spread is very low & $\begin{array}{l}\text { Frequent access to dialysis units and in-centre patients' } \\
\text { clustering can increase the risk of infection spread }\end{array}$ \\
\hline Cost & $\begin{array}{l}\text { In many countries the direct costs of home treatment are substantially } \\
\text { lower than in-centre treatment }\end{array}$ & $\begin{array}{l}\text { Transportation and HCW salaries are the main } \\
\text { determinants of direct costs }\end{array}$ \\
\hline Dialysis schedule & $\begin{array}{l}\text { Flexible schedule in HHD treatment low impact in daily activities } \\
\text { APD usually done during night time }\end{array}$ & $\begin{array}{l}\text { Pre-programmed and fixed schedule } \\
\text { May impact with daily activities }\end{array}$ \\
\hline Telemedicine & $\begin{array}{l}\text { A reliable and secure network connection is necessary } \\
\text { Dialysis treatments should preferentially be done when trained nurses } \\
\text { can supervise devices and interact with patients } \\
\text { Added cost for monitoring devices and other supplies }\end{array}$ & Not necessary \\
\hline Training & $\begin{array}{l}\text { For PD, patients can be trained in } 1 \text { week } \\
\text { For HHD, patients might require longer period (buttonhole puncture, } \\
\text { connection of central venous catheter, treatment of intra-dialysis } \\
\text { symptoms) }\end{array}$ & Not necessary for patients \\
\hline
\end{tabular}

HCW, health-care worker; HHD, home haemodialysis; PD, peritoneal dialysis; APD, automated peritoneal dialysis.

We believe that any effort for a broader use of home treatment would be useful. Early on in the pandemic, SARS-CoV-2 cases were significantly higher among ICHD than in home dialysis patients. But, it is worth pointing out that ICHD patients are exposed to both individual and community factors, and there are reports showing that transmission mitigation was successfully obtained within HD units following the adoption of stringent infection control protocols and procedures [23].

\section{Conclusions}

Policymakers and nephrologists should prioritize home treatments with the aim to reduce the burden on, and to prevent infection spread in, hospitals and crowded dialysis centres. The more patients we have on home treatment programs, the lower the pressure on hospitals in case of a new pandemic (Table 1). However, despite its advantages, PD is still under-prescribed in many countries [24]. For instance, in Italy less than $10 \%$ and in the US only 7.7\% of patients are on PD, while highest proportions are reached in Hong Kong (82\%), Mexico (51\%), and New Zealand (30\%). The COVID-19 pandemic has taught us a lesson: we have to be prepared, well organized, and actively promote home treatment, so as to reduce infection spread and disease burden.

\section{Conflict of Interest Statement}

The authors have no conflicts of interest to declare. 


\section{Funding Sources}

No funding was provided for this article.

\section{Author Contributions}

G.B. provided the idea, and G.B. and G.R. drafted the manuscript. G.B., G.R., and F.A. revised the manuscript and wrote the final version.

\section{References}

1 Reperant LA, Cornaglia G, Osterhaus AD. The importance of understanding the human-animal interface: from early hominins to global citizens. Curr Top Microbiol Immunol. 2013;365:49-81.

2 World Health Organization. Coronavirus disease (COVID-19) pandemic. 2020.

3 Cheng Y, Luo R, Wang K, Zhang M, Wang $Z$, Dong L, et al. Kidney disease is associated with in-hospital death of patients with COVID-19. Kidney Int. 2020 May;97(5):82938.

4 Karopadi AN, Mason G, Rettore E, Ronco C. Cost of peritoneal dialysis and haemodialysis across the world. Nephrol Dial Transplant. 2013 Oct;28(10):2553-69.

5 Rigoni M, Torri E, Nollo G, Zarantonello D, Laudon A, Sottini L, et al. Survival and timeto-transplantation of peritoneal dialysis versus hemodialysis for end-stage renal disease patients: competing-risks regression model in a single Italian center experience. J Nephrol. 2017 Jun;30(3):441-7.

6 Wong B, Ravani P, Oliver MJ, Holroyd-Leduc J, Venturato L, Garg AX, et al. Comparison of patient survival between hemodialysis and peritoneal dialysis among patients eligible for both modalities. Am J Kidney Dis. 2018 Mar; 71(3):344-51.

7 Wares JR, Lawson B, Shemin D, D'Agata EM. Evaluating infection prevention strategies in out-patient dialysis units using agent-based modeling. PLoS One. 2016;11(5):e0153820.

8 Jadoul M, Bieber BA, Martin P, Akiba T, Nwankwo C, Arduino JM, et al. Prevalence, incidence, and risk factors for hepatitis $\mathrm{C}$ vi- rus infection in hemodialysis patients. Kidney Int. 2019 Apr;95(4):939-47.

9 Chen TH, Wen YH, Chen CF, Tan AC, Chen YT, Chen FY, et al. The advantages of peritoneal dialysis over hemodialysis during the COVID-19 pandemic. Semin Dial. 2020 Sep; 33(5):369-71.

10 International Society for Peritoneal Dialysis. Strategies regarding COVID-19 in PD patients. 2020.

11 Wilkie M, Davies S. Peritoneal dialysis in the time of COVID-19. Perit Dial Int. 2020 Jul; 40(4):357-8.

12 Quintaliani G, Reboldi G, Di Napoli A, Nordio M, Limido A, Aucella F, et al. Exposure to novel coronavirus in patients on renal replacement therapy during the exponential phase of COVID-19 pandemic: survey of the italian society of nephrology. J Nephrol. 2020 Aug;33(4):725-36.

13 Xiong F, Tang H, Liu L, Tu C, Tian JB, Lei CT, et al. Clinical characteristics of and medical interventions for COVID-19 in hemodialysis patients in wuhan, China. J Am Soc Nephrol. 2020 Jul;31(7):1387-97.

14 Cecile C, Florian B, Carole A, Clemence B, Philippe B, Francois C, et al. Low incidence of SARS-CoV-2, risk factors of mortality and the course of illness in the french national cohort of dialysis patients. Kidney Int. 2020 Aug 25; 98(6):1519-29.

15 COVID-19 Treatment Guidelines Panel. Coronavirus disease 2019 (COVID-19) treatment guidelines.

16 Perna AF, Capolongo G, Trepiccione F, Simeoni M, Zacchia M, Ingrosso D. COVID-19, low-molecular-weight heparin, and hemodialysis. Kidney Blood Press Res. 2020;45(3): 357-62.

17 Karopadi AN, Mason G, Rettore E, Ronco C. The role of economies of scale in the cost of dialysis across the world: a macroeconomic perspective. Nephrol Dial Transplant. 2014 Apr;29(4):885-92.

18 Agarwal S, Wilkie M. Remote patient management in peritoneal dialysis: opportunities and challenges. Contrib Nephrol. 2019;197: 54-64.

19 Jeffs L, Jamieson T, Saragosa M, Mukerji G, Jain AK, Man R, et al. Uptake and scalability of a peritoneal dialysis virtual care solution: qualitative study. JMIR Hum Factors. 2019 Apr 16;6(2):e9720.

20 Scarpioni R, Manini A, Chiappini P. Remote patient monitoring in peritoneal dialysis helps reduce risk of hospitalization during covid-19 pandemic. J Nephrol. 2020 Aug 11;12.

21 Keesara S, Jonas A, Schulman K. Covid-19 and health care's digital revolution. $\mathrm{N}$ Engl J Med. 2020 Jun 4;382(23):e82.

22 UK Renal Association. COVID-19: checklist and guidance for management of peritoneal dialysis programmes.

23 La Milia V, Bacchini G, Bigi MC, Casartelli D, Cavalli A, Corti M, et al. COVID-19 outbreak in a large hemodialysis center in lombardy, Italy. Kidney Int Rep. 2020 Jul;5(7):1095-9.

24 Briggs V, Davies S, Wilkie M. International variations in peritoneal dialysis utilization and implications for practice. Am J Kidney Dis. 2019 Jul;74(1):101-10. 